\title{
Politica Industrial y Pequeñas y Medianas Empresas: el Caso Argentino
}

Marina Schenkel ${ }^{(*)}$

Resumen: Este trabajo examina el caso de las políticas argentinas para las pequeñas y medianas empresas (PyMEs). Se trata de políticas a las que se les reconoce la capacidad de suplir funciones de fomento y de coordinación que los automatismos del mercado no garantizan. Un elemento de notable interés que presenta el caso argentino es la persistencia de la estructura productiva e inclusive de las exportaciones de la Nación, no obstante las fluctuaciones macroeconómicas tan importantes de los últimos años. La conclusión es que parece probable que el incontestable y reciente éxito de la gran industria en Argentina - que incluye a las industrias más dinámicas - se apoye en relaciones verticales que éstas han instaurado con pequeñas empresas en competencia entre sí Aunque particularmente el éxito de la agroindustria podría demostrar que la política industrial ha sido irrelevante, la estrategia desarrollada por las grandes empresas tanto nacionales como multinacionales y por algunas nuevas figuras empresariales, ha constituido un cabal ejemplo de capacidad de coordinación en un enfoque sistémico. Se ha cerrado la época de los grandes éxitos y fracasos de las políticas estratégicas, y las ambiciones de los tiempos del ISI en Argentina quedan por el momento relegadas, por lo que cabe aquí registrar una correspondiente reducción de la escala y de los objetivos de las políticas industriales. Además los patterns de fondo de las especializaciones productivas, condicionadas por dotaciones e interdependencias consolidadas a través de las experiencias de desarrollo (y de crisis), aparecen difícilmente alterables en un horizonte breve. Sin embargo, las conexiones activadas por las hileras y, sobre todo, la amplitud del tejido industrial de PyMEs potencialmente vitales, aunque expuestas al riesgo de ser afectadas por los procesos derivados de la globalización, parecen condicionar la difusión del bienestar y la misma cohesión social.

Palabras-clave: Politica Industrial, PyMEs, Argentina.

Abstract: This paper examines the policies for Small and Medium Enterprises (PyMEs) in Argentina. These type of policies still are recognized as capable to develop and create linkage effects not brought about by market mechanisms. A particularly interesting feature of the Argentinean case is the fact that the productive and export structures persist despite the pronounced macroeconomic fluctuations of the last years. The conclusion is that it seems likely that large manufacturing enterprises performance in recent times - the most innovative segment included - is based on vertical relations with SMEs which compete between themselves. Even if the agroindustry success could demonstrate that industrial policy is irrelevant, on the contrary the national and multinational large enterprises strategy, as well as the one displayed by new enterpreneurial figures, have been a notable exemple of coordination capacity in a systemic approach. The epoch of the great success or defeats of strategical policies is over, and the ambition of the Isi (Import Substitution) are for the moment put aside: therefore both the scale and objectives of industrial policies are reduced. Moreover the fundamental patterns of productive specializations, restrained by resources and ties consolidated though development and crisis experiences, are not easily changed in the short run. However the relations established by clusters and the size of the industrial base formed by potentially viable, even if exposed to the risks deriving from globalization processes, SMEs seem to condition welfare diffusion, and social cohesion itself.

Keywords: Industrial Policy, SMEs, Argentina.

(*) Profesor cathedratico de Economia Aplicada, Facultad de Economia, Universidad de Udine, Itália. E-mail: marina. schenkel@uniud.it. Recebido em 12.5.09 e aceito em 20.8.09. 


\section{INTRODUCCIÓN}

Este trabajo examina el caso de las políticas argentinas para las pequeñas y medianas empresas (PyMEs) e intenta procurar ejemplos exportables y de ofrecer posibles indicaciones que deben tenerse en cuenta para evitar errores cometidos en otros países. Se trata de políticas a las que aún se les reconoce la capacidad de suplir funciones de fomento y de coordinación que los automatismos del mercado no garantizan en esta fase histórica, en que el eclipse de la idea de la gran empresa pública o de los "campeones nacionales" y la dudosa capacidad de influenciar las decisiones de la gran empresa transnacional parecen hacer inviables otras herramientas ya utilizadas para impulsar el desarrollo y la especialización, que, nuevamente, se presentan como condicionadados fundamentalmente por la asignación de recursos naturales y culturales y de capital humano de los diferentes contextos.

La Argentina es un país rico en recursos naturales, relativamente despoblado, y caracterizado por la dualidad (y en algunos períodos por el conflicto) entre la ciudad y el campo; la alta densidad de pequeñas y medianas empresas parece acercarla a Italia. El propósito de la evaluación de los límites y del espacio de las policies nacionales puede, sin embargo, revelar en qué medida la dimensión mundial, en la cual se despliegan la movilidad de los capitales y la proyección estratégica de los grandes operadores, hace de la diversidad de los modelos nacionales un elemento de detalle secundario, o, en cambio, cuánto la persistencia de las especificidades locales de los factores y de los agentes, necesariamente menos móviles, sigue siendo significativa al momento de medir la capacidad de valorar y acrecentar la ocupación, en otros términos la vitalidad de las economías nacionales y regionales.

En esta perspectiva, un elemento de notable interés que presenta el caso argentino es un hecho particularmente destacado por varios estudios (FERNÁNDEZ BUGNA, PORTA 2007; LAVOPA, 2007; CEP 2005b): la persistencia de la estructura productiva e inclusive de las exportaciones de la Nación, no obstante las fluctuaciones macroeconómicas tan importantes de los últimos años. A primera vista el impacto de la política industrial parecería de escasa importancia, pero también sería inesperadamente modesto el impacto de las condiciones macroeconómicas internas, de la dinámica de los precios internacionales y, en general, de las nuevas configuraciones del mercado mundial en la estructura productiva y en la especialización comercial. Se tratará entonces de señalar la importancia que logre alcanzar la política industrial, no tanto porque pueda determinar un cambio estructural, que ni siquiera se ha dado, sino por el rol que puede desempeñar en relación a la recalificación de la preexistente estructura productiva y, sobre todo, a la conservación del tejido de $\mathrm{PyMEs}^{(1)}$ en un sistema económico afectado por pronunciadas dinámicas, ya sean de signo positivo o negativo, del PBI, y por el drástico cambio de los términos de intercambio en el comercio internacional.

(1) En la literaturay en las estadísticas disponibles bastante frecuentemente se distingue entre PyMEsy Microempresas (Mini), inferiores a 5 empleados. Otras definiciones utilizadas con fines específicos se refieren al facturado o a las exportaciones. En el presente trabajo no se distingue dentro del total que se entiende, de todos modos, formado por todas las empresas que no son "grandes", es decir superiores a 250 empleados. 
Los siguientes parágrafos entrarán en el detalle de los hechos recién descriptos, haciendo referencia a la literatura en materia y en la medida de lo posible, a la poco satisfactoria documentación estadística oficial disponible. ${ }^{(2)}$

Dado que la pronunciada inestabilidad macroeconómica se refleja también en la inestabilidad política, será necesaria una constante referencia a la compleja historia reciente $^{(3)}$ con el fin de realizar una correcta evaluación de la influencia de la política industrial sobre las pequeñas y medianas empresas y disponer de elementos de comparación con la experiencia de otros países.

\section{LA ESTRUCTURA INDUSTRIAL, EL LEGADO DE LOS AÑOS 90 Y LA CRISIS}

El decenio de los años 90 (el período de la convertibilidad) representa la fase crucial en la cual toma forma la estructura industrial que parece persistir aún hoy. A través de la combinación de un cambio sobrevaluado, y de la completa apertura de la economía a los flujos de bienes y servicios y de capital, se obtienen las condiciones para un rápida, y en algunas casos traumática, racionalización y modernización del aparato productivo existente. El factor trabajo resulta relativamente más caro a nivel internacional, aunque con escaso poder adquisitivo a nivel interno. El factor capital resulta, en cambio, relativamente menos costoso, sea por la baja tasa de interés, como por la paridad peso-dólar. Al mismo tiempo están desfavorecidas las industrias productoras de bienes tradable y favorecidas las producciones de bienes nontradable y los derivados de recursos naturales propios del país. Es en estos sectores que se desarrollan las inversiones externas, efectuadas predominantemente a través de fusiones y compras, más que con inversiones greenfields.

Las inversiones asumen sobre todo la forma de importaciones de maquinarias y plantas, lo que si por un lado implica la falta de desarrollo de capacidades endógenas de innovación, sobre todo en el campo de Investigación y Desarrollo (ANLLÓ, LUGONES, PEIRANO, 2007), por el otro conduce a un aumento notable de la productividad media del trabajo (+51\% entre 1991 y 1998), que se mantiene inalterado, aunque las tasas de incremento serán menores ${ }^{(4)}$ durante los posteriores acontecimientos económicos.

Es en este período que se sientan las bases de lo que se puede definir el éxito más espectacular de la economía argentina: la "fuga hacia delante" de la agricultura. La introducción de la soja transgénica, unida al uso del herbicida al que es resistente - el glifosato de sodio - y a la técnica de la siembra directa, por la cual el terreno no debe ararse preventivamente, es el más claro ejemplo de este extraordinario proceso de innovación.

Cabe notar que en Argentina se pone en venta la semilla de la planta transgénica en 1996, dos años después que en los EE.UU., con evidente anticipación respecto a los

(2) Hasta hoy, en efecto, no están disponibles los resultados del Censo Nacional Económico del 2004-2005. Cf. Lavopa (2007), también por los posibles efectos de distorsión en el cálculo del PBN por la falta de actualización del registro de empresas utilizado.

(3) Para una síntesis de la historia del desarrollo argentino hasta la crisis, ver Bianchi (2002).

(4) Schorr (2007) se refiere sin embargo a un aumento del 21\% entre el cuarto trimestre del 2001 y el cuarto trimestre del 2004. 
demás Países. En estos años, que bien podrían llamarse pionerísticos, el financiamiento es sostenido sobre todo por los bancos públicos, que en Argentina nunca han dejado de existir, a pesar de la oleada de privatizaciones, aunque a partir de 1998, paralelamente al empeoramiento de la situación económica, crece el rol de las bancas privadas y por ende el endeudamiento del campo. ${ }^{(5)}$

Es sumamente interesante notar que, ante la carencia de una efectiva iniciativa de la política industrial, las grandes empresas privadas, en parte trasnacionales (Monsanto, Syngenta, Bayer), van creando una red de Centros de Servicios para difundir en toda la Argentina las ventas del paquete (semillas, herbicidas y fertilizantes), cuya capacidad de penetración hacia las llamadas economías regionales, más allá de los confínes de la Pampa húmeda (la llanura central que constituye los territorios de las provincias de Buenos Aires, Córdoba, Santa Fe y Entre Ríos), está además determinada por la asistencia técnica y financiera prestada a los agricultores.

La segunda transformación crucial que ocurre en aquellos años concierne a la figura del contratista. Este actor, que siendo un trabajador autónomo por cierto no puede definirse nuevo, dado que que en el campo siempre ha habido quien presta su trabajo a contrato, constituye un elemento innovador por el hecho de que, sin poseer necesariamente la tierra ("agricultores sin tierra")(6), desempeña un rol empresarial, es decir, se encarga de la gestión de la producción agrícola, asumiendo el riesgo correspondiente. Aunque éste no es el ámbito para adentrarnos en la descripción de las características de estos empresarios ${ }^{(7)}$, es necesario recordar que se trata de personas de origen heterogéneo, en parte "gringos" de reciente ascendencia europea y por tanto ajenos a la tradicional oligarquía criolla, de antigua descendencia española, que se remonta a la época colonial.

Se trata de empresas "transhumantes", no ligadas a un territorio específico, que se convierten en "vectores del cambio tecnológico" (BISANG, 2007). Se crean estructuras de $\operatorname{red}^{(8)}$ que incluyen empresas de transformación e infraestructuras ferroviarias y portuarias organizadas a partir de un vértice, que, aun siendo muy restringido, domina algunas principales tecnologías ${ }^{(9)}$, por lo que se establecen fuertes conexiones entre todos

(5) Bisang (2007), sobre la base de una apasionante reconstrucción de los acontecimientos, llega a la conclusión de que fue el endeudamiento de los propietarios a obligarlos a la innovación, y a los bancos a sostenerlos.

(6) Según los datos del Censo Agrícola del 2002, los 2/3 de los trabajos agrícolas están desarrollados por sujetos diferentes a los propietarios de la tierra (BISANG, 2007).

(7) Descripciones esclarecedoras, también con el fin de comprender otros aspectos de la historia y de la sociedad argentina, se pueden encontrar por ejemplo en Bisang (2007) y Hernández (2007).

(8) Para describir estas configuraciones en red ha sido utilizado el concepto de "trama productiva" (BISANG, GUTMAN, 2003), cuyas afinidades con el keiretzu japonés no han sido aún objeto de investigación.

(9) Los pools de siembra, fondos de inversión en actividades administradas en general por ingenieros agrónomos, asumen un rol innovador esencial con este propósito ("Cualquier persona que tenga una buena idea y buen management puede sembrar"). Es emblemática la figura de Grobocopatel, que se define como «pastor de la sociedad del conocimiento» y así describe a su empresa: "Nuestra empresa es una red de producción. Estamos sembrando 80.000 hectáreas de agricultura, de commodities, en la Argentina; 20.000 hectáreas en Uruguay y 6.000 in Paraguay. Pero estas siembras no las hacemos solos; las hacemos en distintas sociedades con los dueños de la tierra, con proveedores de servicios, con proveedores de insumos, a través de integraciones de distinto tipo, muy flexibles (herbicidas, pesticidas, semillas etc.). (...) Hoy, en la red de producción, las 8000 hectáreas se hacen con cinco ingenieros agrónomos, pero con doce PyMEs de gerenciamiento agrícola 
los actores involucrados, en el sentido que el éxito de una empresa depende del de todas las demás (ANLLÓ, LUGONES, PEIRANO, 2007).

El aumento de los rendimientos y de la superficie abarca a toda la agroindustria, con recaídas no sólo en los sectores industriales involucrados, sino sobre los equilibrios macroeconómicos, como se verá en el páragrafo siguiente dedicado a los desarrollos más recientes de la situación agraria.

En este período se desarrolla también, con una estructura de red en algunos aspectos análoga, la industria automovilística, sobre todo por los esfuerzos de política industrial coordinados entre Países (sustancialmente Argentina y Brasil) miembros del Mercosur ${ }^{(10)}$.

Como consecuencia de los procesos recién mencionados, la especialización de la Argentina resulta orientada hacia algunos sectores específicos, a los cuales se dirigen también las inversiones externas directas (IEDs), relacionadas a la explotación de los recursos naturales, agrícolas y no (petróleo, gas y derivados, minería) y al desarrollo de servicios públicos y del terciario avanzado (inmobiliario, financiero, salud, etc.).

Procesos de concentración, y por lo tanto de reducción de la cuota de PyMEs, parecen acompañar a estas dinámicas, pero, por la carencia de información antes mencionada, resulta difícil documentarlos.

A partir de 1998 se verifica la coincidencia de una serie de circunstancias externas desfavorables, entre las cuales se destacan la devaluación del real brasileño y la caída de los precios internacionales de las materias primas. Se incia un violento proceso de ajuste, requerido por los desequilibrios generados por el

modelo de la convertibilidad, que, como es sabido, se manifiestan sobre todo en un insostenible déficit de las cuentas externas. En el siguiente quinquenio, hasta el segundo semestre del 2002 en que la economía sale de la recesión, se suceden acontecimientos caóticos e inclusive dramáticos, cuya reconstrucción no corresponde emprender en este trabajo, que culminan con la devaluación y el default (diciembre del 2001) ${ }^{(11)}$.

En el mismo período se asiste a una importante caída del sector manufacturero $(-18 \%)$ y en la costruccion (-6\%), mientras que el sector agricultura y cría $(+1.2 \%)$ y, sobre todo, el minero (+8\%) mantienen una dinámica positiva (FERNÁNDEZ BUGNA, PORTA 2007). Son desfavorecidas, sobre todo, las empresas dirigidas al mercado interno y/o a los mercados de los países vecinos, y entre éstas particularmente las PyMEs. En éstas colapsan la ocupación y las inversiones, pero las disminuciones no son tan marcadas en las otras: los datos sobre la mortalidad de las empresas y sobre la ocupación del Ministerio de Trabajo (MTEySS) (Estadísticas del Observatorio de la Dinámica de Empresas y Empleo) testimonian no sólo la gravedad (mortalidad +50\%) sino también

asociadas; cada una de ellas, son empresas separadas, tienen dos o tres ingenieros agrónomos, van a riesgo con nosotros y son socios nuestros en la producción agrícola; contratos, PyMEs para labores, PyMEs para cosechas. Finalmente somos quince personas físicas y 135 empresas contratadas, que emplean alrededor de 480 personas directamente y mil quinientos en forma indirecta" G. Grobocopatel", Red social del conocimiento, documento del 2004, citado en Hernández (2007).

(10) Para más detalles, ver el Apéndice.

(11) Una reconstrucción detallada e informada, si no imparcial a menudo (auto)irónica, en Levy Yeyati y Valenzuela (2007). 
la heterogeneidad del shock, en el sentido que no todo el tejido productivo colapsa en igual medida (KANTIS, FEDERICO, 2007). Al mismo tiempo, debido al crecimiento de la desempleo, en muchos casos se provoca una reducción cospicua del salario nominal.

A partir de la devaluación la situación precedente, en lo que respecta a los precios relativos, registra un neto revés: el precio de las importaciones (y por lo tanto de los bienes de capital) se alza, y se reduce el salario real.

\section{LA RESURRECCIÓN}

La fase de recuperación, tan rápida y pronunciada como la de la crisis, ha llevado la Argentina a alcanzar en el 2005 el nivel del PBI pre-crisis, con tasas de crecimiento record entre los países occidentales, superiores al 8\% anual. A partir del 2003 hasta hoy, la época de los déficit es reemplazada por la de los superávit gemelos, ayudada, pero no únicamente determinada, por la explosión de los precios de las materias primas.

Es la industria manufacturera la primera protagonista de la reactivación, con tasas de crecimiento en la producción que llegan a obtener ya en el 2005 los niveles máximos alcanzados en 1994. Las PyMEs van a la vanguardia en el aumento de los montos facturados (FUNDACIÓN OBSERVATORIO PYME, 2006), en la recuperacion de la ocupación y, obviamente, en la natalidad de empresas: Microempresas $+27 \%$, PyMEs $+10.5 \%$. $\mathrm{Su}$ aporte a la dinámica exportable es modesto en términos absolutos: en el 2005 el porcentaje de PyMEs que pueden definirse exportadoras es sólo del 10\% (FUNDACIÓN OBSERVATORIO PYME, 2006).

Sin embargo, las exportaciones de las PyMEX (Pequeñas y Medianas Empresas Exportadoras) son superiores a las de las grandes, sea en incremento del valor total como, sobre todo, del precio medio: $\$ 353$ por tonelada para las grandes, $\$ 608$ para las medianas, \$943 para las pequeñas (CEP, 2005a).

Aunque - como otros han destacado (LAVOPA, 2007) - no estemos en condiciones de ir más allá de simples suposiciones escasamente respaldadas por datos puntuales, es posible que los procesos de desverticalización y tercerización de los años 90 (los años de las reformas) hayan sentado las bases para la posterior integración de una trama de PyMEs dinámicas en la red cuyo liderazgo pertenece a las grandes empresas exportadoras, o que el impacto sobre el Valor Agregado de las PyMEs y el sostenimiento que ellas brindan a la ocupación sean efectivamente modestos, debido a sus bajos niveles de productividad. Por lo cual, la ocupación creada sería en realidad una forma de disguised unemployment. En espera de posteriores datos estadísticos, y más que nada de las publicaciones de los resultados del Censo Nacional Económico 2004-2005, las mejores y más estudiadas estadísticas a disposición resultan ser las derivadas de la Contabilidad Nacional. Según estos datos, los sectores que exhiben tasas de incremento mayores son los que ya fueron golpeados por la crisis, y aunque ello no resulte sorprendente, es interesante resaltar que, en términos temporales, son éstos los sectores que reaccionan primeros a las condiciones macroeconómicas modificadas, pudiendo contar con una abudante capacidad productiva inutilizada. Se trata del textil y vestidos, mecánica (exclusas las maquinarias), materiales para la construcción, aparatos audio y video, maquinarias e 
implantes eléctricos. Sin embargo, a pesar de su brillante comportamiento post-2002, estas industrias no han logrado recuperar los niveles máximos alcanzados en el pasado, antes de los años 90. La excepción en este caso es la industria automovilística que, aunque reactivándose recién en el 2004, y por lo tanto, atrasada respecto a los otros sectores, estuvo en condiciones de volver a alcanzar en el 2006 los niveles máximos obtenidos en 1997 (FERNÁNDEZ BUGNA, PORTA, 2007). Las industrias que, en cambio, consolidan su primado son las que se habían afirmado en los años de la liberalización y que habían caído menos en los años de la recesión: las industrias productoras de commodities y basadas en la explotación de recursos naturales; metales, química de base, papel, combustible y alimenticios. La comparación entre la estructura industrial de 1998 y la del 2005 revela que no han habido cambios estructurales significativos: éstos ocurrieron antes, con las reformas que han determinado el eclipse del modelo de sustitución de importaciones y el declive relativo de la industria manufacturera. Confirma esta hipótesis el interesante ejercicio realizado por Lavopa (2007), que compara el actual período con el tiempo en que hubo otra resurrección, luego de la crisis mexicana (el tequilazo) de 1995. En ambos casos resulta que las industrias que mayormente han contribuído al proceso son las mismas, en proporción muy similar y con tasas de desarrollo comparables ${ }^{(12)}$. Esto vale también en lo que atañe a las industrias líderes del proceso de desarrollo genuino, posterior a la fase de recuperación de los niveles anti-crisis, es decir la construcción, el comercio, las telecomunicaciones, la intermediación financiera e inmobiliaria, los alimenticios, el transporte. Signo de la continuidad con el pasado más reciente y de la imposibilidad de retorno a un tiempo más lejano, parece ser la no lograda sustitución de las importaciones, no obstante el tipo de cambio fuertemente subvaluado (FERNÁNDEZ BUGNA, PORTA, 2007). Las PyMEs son las que más se resienten con la situación: según una investigación realizada en el 2005 por el Observatorio (2006), el 40\% se siente amenazada por la competencia externa. Tambièn, segùn otras fuentes (CEP 2005b), algunas industrias han sido protagonistas de un cierto proceso de substitución de las importaciones (papel y celulosa, química para la agricultura, acero, materiales para la construcción, alimentos y bebidas).

Un elemento posterior que refuerza la estructura productiva preexistente es la reactivación de las inversiones, y de las inversiones externas directas (IED), en las proporciones inter e intra industriales ya experimentadas antes de la crisis (FERNÁNDEZ BUGNA, PORTA, 2007). España y Estados Unidos siguen ocupando los primeros puestos, mientras aumentan las inversiones por parte de Brasil.

La balanza comercial industrial es positiva desde el 2002, después de un decenio de saldos negativos (Fernández Bugna y Porta 2007). Las industrias cuyas exportaciones superan a las importaciones son: alimentos, madera, refinerías de petróleo, pieles y cueros y metales comunes. Al menos hasta el 2006 es la Manufactura de Origen Industrial (MOI), en que asume un peso siempre creciente la industria automovilística, la que confiere el mayor aporte al gran crecimiento de las exportaciones $(+47.50 \%$ entre

(12) Es interesante la comparación entre los dos períodos también en lo que se refiere a la creación de puestos de trabajo: mientras que en el post-Tequila prevalece la gran empresa, en el post-convertibilidad asumen un rol preponderante las pequeñas y, sobre todo, las microempresas y los micro emprendedores (LAVOPA, 2007). 
1996 y 2006), respecto a la Manufactura de Origen Agrícola (MOA), a los combustibles y energía y a los productos primarios (Fernández Bugna y Porta 2007). En el conjunto aunque la estructura de las exportaciones no se modifique de manera significativa, se asiste a un importante cambio del signo y de las destinaciones de los flujos: aumentan y se convierten en saldo positivo aquellos destinados a los países del norte y del este (China e India), convirtiéndose en saldo negativo los destinados a Brasil.

En el crecimiento generalizado que sigue a la devaluación aumentan los beneficios para todas las empresas, sea por la compresión de los salarios como, en muchos casos, por la liquidación de las deudas y de los pasivos ${ }^{(13)}$. Dado que el aumento es generalizado, se mantienen los diferenciales inter e intra industriales heredados del período precedente (FERNÁNDEZ BUGNA, PORTA, 2007).

\section{EMPRESAS Y EstAdo EN UN AMBIENTE INESTABLE}

La característica continuidad, no obstante el contexto turbulento, del marco de especialización industrial delineado en el párrafo anterior, vuelve a plantear la cuestión central del rol y la incidencia de la política industrial. Y, sobre la base del examen de casi veinte años, parece delinearse la conclusión ya anticipada al inicio que la política no ha incidido en el sentido de provocar un cambio estructural, sino más bien en el de favorecer la persistencia de un diferenciado tejido de PyMEs junto a la gran empresa. Este páragrafo tratará de reconstruír las líneas generales de las intervenciones, con algunas evaluaciones de conjunto extraídas de la literatura en la materia ${ }^{(14)}$. En el período de substitución de importaciones (Industrialización por Substitución de Importaciones, ISI) las politicas, basadas sobre todo en la filosofia de la reserva de mercado, eran sustancialmente de índole proteccionista, dirigidas a las promociones en el mercado interno, y por lo tanto, "viejas", es decir, sectoriales y frecuentemente finalizadas a promover determinadas regiones o provincias, cuando no empresas específicas (generalmente grandes empresas locales o multinacionales) (BARUJ, RAMOS, KOSAKOFF, 2007). Sin embargo, ya a partir del 1987-1988 se comienza a cambiar la dirección en sentido "horizontal", con el fin de estimular las exportaciones y mejorar las habilidades tecnológicas y de management, con énfasis en las PyMEs. Lo cual conlleva el resultado que las políticas, aun ganando en extensión debido a que aumenta el número de empresas alcanzado, resultan de impacto macroeconómico limitado, sobre todo en relación a la fuerza de las dinámicas del cambio, y en general, de los precios relativos a nivel internacional.

En el marco general, aun predominando el planteamiento "horizontal" (15), quedan huellas del pasado sectorialismo, sobre todo en lo que respecta a los incentivos a las inversiones, y de políticas verticales que se remontan a los años 90 dirigidas a la promoción de "nuevos" recursos naturales (minas de metales, forestas) o de la industria automovilística al interno del Mercosur y, más recientemente, de la industria del software.

(13) Lo que se ha denominado "pesificación asimétrica" significó que los deudores en dólares se encontraron devolviendo, el mismo importe en pesos, salvo una compensación al banco a cargo del Estado y, por lo tanto, del contribuyente.

(14) Para una descripción detallada de los diferentes institutos y programas, se reenvía al Apéndice.

(15) La Argentina parece compartir con Chile y Uruguay la mayor orientación hacia políticas "horizontales" (PERES, 2006). 
Por otro lado, caracterísitico de la situación argentina es que instrumentos concebidos para compensar la pérdida de competitividad de las empresas provocada por la sobreevaluación de la moneda nacional en el período de la convertibilidad, y medidas de emergencia para salvar a algunas industrias (tabaco, bienes de capital, maquinas agrícolas) del colapso de dicho régimen en el 2001-2002, permanezcan aún cuando las condiciones macroeconómicas y de los mercados internacionales sean diferentes y hasta opuestas.

Parecería que otra característica típicamente argentina es la escasa importancia de las políticas locales y el peso preponderante de los Programas Nacionales, cuyas idea y gestión se concentran en la Capital Federal. Sin embargo es constante, a partir de los años 90, la atención de la política de desarrollo regional hacia los conceptos de cluster, "casi distrito", "red empresarial local", "área económica local ", usados por varios autores en el estudio de algunas áreas del centro del País, y especialmente de la ciudad industrial de Rafaela ${ }^{(16)}$.

A pesar de la impronta centralista, la presencia de estratificaciones de períodos (y Gobiernos) diferentes llevan a notables fragmentaciones y sobreposiciones de iniciativas que pueden inducir a pensar en la falta de una "idea país". Cuánto esto se traduzca en ineficiencia o en incoherencia, y cuánto en flexibilidad frente a shocks de origen externo, o por lo menos en menor riesgo de tomar calles sin salida (como, en cambio, ha revelado ser la institucion del currency board) es una cuestión que por ahora resulta escasamente percibida en el debate que actualmente se desarrolla en sedes académicas y no académicas. Más en general, la falta de una evaluación de las políticas, sea en sede oficial como en la literatura científica, va acompañada de escasez de transparencia y de detalle de informaciones sobre la utilización de los fondos y de los resultados obtenidos. Queda de todos modos la tentación de parte de los economistas que siguen desde hace mucho tiempo el argumento, de expresar un juicio negativo y la necesidad de un cambio radical (FERNÁNDEZ BUGNA, PORTA, 2007).

Dado que el contacto con el sector privado es requerido por las diferentes instituciones públicas sólo en el momento de la decisión sobre la oportunidad de lanzar una iniciativa, son poco numerosos los ejemplos en los cuales la colaboración continúa con la participación activa de las empresas en el diseño de los proyectos mismos (BARUJ, RAMOS, KOSAKOFF, 2007). En general las empresas, aún declarando en altos porcentajes que efecúan investigación interna y que han introducido innovaciones de producto y de proceso en ocasión de las Encuestas de Innovación efectuadas por un cierto numero de años por el Instituto Nacional de Estadísticas (INDEC), no aparecen insertas en relaciones de cooperación tecnológica con otras empresas y entes de investigación públicos o privados, ni parece que hubiera una demanda explícita de servicios tecnólogicos y de asistencia para la inversión (ANLLÓ, LUGONES, PEIRANO, 2007). Análogamente, las variables institucionales (cooperación con otras empresas o instituciones, conocimientos y uso de los programas de promoción de las exportaciones) no parecen haber influenciado en el éxito de las PyMEs exportadoras (MILESI, MOORI, ROBERT, YOGUEL, 2007). Los fondos comprometidos resultan además generalmente de limitado importe y

(16) Una breve reseña crítica y una discusión de los casos en Kulfas, Duarte y Schorr (2008). 
concentrados en pocas instituciones. A lo que cabe agregar la característica inestabilidad de las instituciones mismas, en cuanto a la dotación financiera y al personal empleado. Esta situación impide el afianzamiento de las habilidades, del conocimiento y de las relaciones, y la viabilidad misma de las iniciativas.

\section{EL PROBLEMA DEL CAMPO}

A pesar de la dificultad que implica la descripción de una realidad aún demasiado reciente para un analisis imparcial, y en ausencia de datos suficientemente desagregados y actualizados al 2008, no es posible eludir una mención, aunque impresionista, a una de las cuestiones más complejas e interesantes de la situación argentina: el desarrollo de las producciones agrícolas. Éstas, en el lapso de 15 años, se han duplicado en cantidad, mientras que dentro de las mismas, por efecto de la técnica de doble cultivo, la soja junto con el grano han tomado una importancia cada vez mayor. Evolución similar han presentado los productos derivados de la leche, mientras que el desarrollo de la ganadería ha sido positivo, pero no tan brillante.

No sólo las producciones de tipo agro-alimenticio se muestran como la parte más relevante y dinamica de las exportaciones en los últimos años (BISANG, 2007; ASIAIN, $2008)^{(17)}$, sino que - y el dato es verdaderamente nuevo e importante - éstas representan el ejemplo más significativo de innovación y de reorganización en sentido industrial de la economía argentina. Ello nos induce a pensar que no tenga más sentido hablar de la agricultura como de un sector estancado, de bajo valor agregado, al cual se contrapone un sector industrial dinámico e innovador ${ }^{(18)}$. En realidad es la misma división entre Agricultura e Industria y entre Agricultura y Servicios que está en tela de juicio por la evolución reciente de los mercados mundiales y por la revolución técnica y organizativa que ésta ha inducido (SZTULWARK, 2007). Sin adentrarnos en una discusión que trasciende los límites del presente trabajo, los fines que éste se propone requieren qe hagamos otra consideración, y es que precisamente en el sector agropecuario ${ }^{(19)}$ ha

(17) Asiain (2008) calcula que en ausencia de las exportaciones de soja y derivados el superávit de la Balanza Comercial se transformaría en un un déficit grosso modo del mismo orden de grandeza. Si bien lo mismo se podría sostener con relación a cualquier otro producto exitoso (por ej. de la industria automovilística), el razonamiento es sugestivo, y muy común entre viejos y nuevos paladines del campo también más allá de sedes académicas. Bisang (2007) calcula que el porcentaje de agroalimentos (materias primas y bienes manufacturados de origen animal) es siempre superior al $50 \%$ del total de las exportaciones entre el 2001 y el 2005.

(18) A raíz de lo que se afirma en el texto, el debate sigue rotando sobre cuestiones no por cierto nuevas: la thesis de PrebishSinger, o sea el necesario deterioro de los términos de intercambio en contra de los países productores de materias primas, o bien el Dutch Disease.

Cabe en este momento recordar que talvez el primero a hablar de algo como la maldición de los recursos naturales fue Eduardo Galeano (1971, p. 111): "Las trece colonias del norte tuvieron, bien pudiera decirse, la dicha de la desgracia. Su experiencia histórica mostró la tremenda importancia de no nacer importante.

Porque al norte de América no había oro ni había plata, ni civilizaciones indígenas con densas concentraciones de población ya organizada para el trabajo, ni suelos tropicales de fertilidad fabulosa en la franja costera que los peregrinos ingleses colonizaron. La naturaleza se había mostrado avara, y también la historia: faltaban los metales y la mano de obra esclava para arrancar los metales del vientre de la tierra. Fue una suerte".

(19) El término agropecuario se refiere a una configuración típicamente argentina, para la cual sobre la misma tierra se alternan cultivos intensivos con cría extensiva. (BISANG, 2007). 
sido aplicada una política, que podemos definir industrial, de signo negativo, es decir, la gravación a las exportaciones a través de retenciones. Éstas fueron establecidas en marzo del 2002 por un total del 5\% en lo que respecta a los productos industriales y del 20\% para el sector agropecuario y, sucesivamente, a los hidrocarburantes, lo que constituye una medida que equivale a la imposición de una tasa de cambio diversificada para algunas categorías de bienes (ASIAIN, 2008). Para apreciar el efecto sobre la economía del País, bastaría considerar que de esta manera se determina también el precio interno, o de todos modos se obtiene el efecto de desligar la dinámica inflacionaria interna de las fluctuaciones de los precios internacionales. Este dato es de particular importancia en una situación como la actual, en la que el bienestar de la población y, sobre todo, de las cuotas de ella que se encuentran sobre la línea de pobreza, depende esencialmente del precio de la comida y de los combustibles. Por otra parte, desde un punto de vista fiscal, el crecimiento de las entradas ha permitido financiar programas de redistribución del rédito y de restitución de deuda a organismos internacionales ${ }^{(20)}$.

\section{CONCLUSIONES: ¿QUÉ PODEMOS APRENDER DE LA CRISIS Y RESURRECCIÓN DE LA INDUSTRIA ARGENTINA?}

¿Es posible determinar en base al anterior análisis si aún en la "resurrección" permanecen inmutados los problemas de Argentina, que Bianchi (2002) sintetizaba como: crecimiento sin industrialización, apertura sin integración, privatización sin reglas? Dejando de parte los dos últimos puntos, a la luz de las evidencias presentadas y de las consideraciones desarrolladas anteriormente, la cuestión de la falta de industrialización debería de considerarse en una perspectiva diferente.

Asumiendo la pregunta enunciada, la primera conclusión que podemos abordar es que el caso argentino nos vuelve a poner en evidencia que después de la crisis llega la resurrección, y de él se desprende, aunque no tan simplemente, una segunda enseñanza: una determinada estructura industrial — incluyendo ésta, por lo que hemos expuesto, el conjunto de las actividades de origen y transformación agrícola - no sólo puede sobrevivir a la crisis, sino que puede ser la fuerza que determina, o al menos contribuye sustancialmente a la resurrección. Lo cual tiene por corolario que en una situación de oscilación cíclica acentuada, la influencia de la política industrial se ejercita sobre todo hacia la preservación del tejido productivo formado por las pequeñas y medianas empresas, cuyo colapso provocaría de otra manera costos sociales y consecuencias macroeconómicas inaceptables. Además, por efecto de la mayor relevancia de las estructuras

\footnotetext{
(20) El conflicto estalla al inicio del 2008, en oposición al proyecto gubernativo de adecuar automáticamente, y de manera diferencial según los diferentes productos, la imposición fiscal a los aumentos de los precios internacionales. Todas las partes del campo se asocian en la protesta, que toma tonos violentos, tanto que llega a provocar recuerdos desagradables y miedos nunca del todo acallados en los ambientes democráticos del País. Cabe notar que las grandes empresas exportadoras (entre ellas la mayores son Cargill y Bunge Argentina (oleaginosas), LCD Argentina (Dreyfus) (granos), Aceitera General Deheza (oleaginosas) y Molinos del Río de la Plata, Vicentín y Nidera (granos) que efectuaron en el 2007 el 26,6\% de las exportaciones nacionales) y los pools de siembra han evitado los roles de primer plano en esta ocasión, a diferencia de las organizaciones tradicionales (ruralistas) y nuevas (autoconvocados) de tipo asociativo. Al final el Gobierno fue obligado por el voto parlamentario a retirar el proyecto. El caso provoca laceraciones aún abiertas al interno del equipo de Gobierno y del partido de la mayoría (Justicialista, de inspiración peronista). Ver Giarracca, Teubel y Palmisano (2008).
} 
en red que presentan los sectores agroindustrial y mecánico, las PyMEs desempeñan un papel mayormente importante también desde el punto de vista microeconómico. Aunque esta afirmación deberá apoyarse sobre una base de datos estadísticos más congruente y corroborarse con apropiadas investigaciones, parece probable que el incontestable y reciente éxito de la gran industria en Argentina - que incluye a las industrias más dinámicas - se apoye en relaciones verticales que éstas han instaurado con pequeñas empresas en competencia entre sí. Lo que por supuesto no impide que en muchos otros sectores permanezca el dualismo típico de los países no completamente desarrollados (CIMOLI, PORCILE, PRIMI, VERGARA, 2005).

Aunque particularmente el éxito de la agroindustria podría demostrar que la política industrial ha sido irrelevante, en realidad, dando por cierto lo que tal no es totalmente - es decir, que la política no haya favorecido el desarrollo de la transformación tecnólogica de los cultivos ${ }^{(21)}$ - la estrategia desarrollada por las grandes empresas tanto nacionales como multinacionales y por algunas nuevas figuras empresariales, ha constituido un cabal ejemplo de capacidad de coordinación en un enfoque sistémico.

El fracaso del Gobierno en la aplicación de las retenciones demuestra sin embargo que la acción política perseguida por actores privados mientras propende, por su propia naturaleza, a procurar ventajas sectoriales, por otro lado crea tensiones y conflictos que el público debe saber afrontar en un más amplio contexto de fines compartidos, adoptando instrumentos reguladores e implementando incentivos que vayan más allá de la simple imposición fiscal (BISANG, 2007; ANLLÓ, LUGONES, PEIRANO, 2007).

Se ha cerrado la época de los grandes éxitos y fracasos de las políticas estratégicas. Al final - y es esta la la tercera conclusión que nos parece posible obtener - las ambiciones de los tiempos del ISI en Argentina quedan por el momento relegadas, por lo que cabe aquí registrar una correspondiente reducción de la escala y de los objetivos de las políticas industriales. Además los patterns de fondo de las especializaciones productivas, condicionadas por dotaciones e interdependencias consolidadas a través de las experiencias de desarrollo (y de crisis), aparecen difícilmente alterables en un horizonte breve, como demuestra, por ejemplo, el echo que en la maquinaria la que más se expande es la agroindustrial.

Sin embargo, las conexiones activadas por las hileras y, sobre todo, la amplitud del tejido industrial de PyMEs potencialmente vitales, aunque expuestas al riesgo de ser afectadas por los procesos derivados de la globalización, parecen condicionar la difusión del bienestar y la misma cohesión social. Los recursos humanos, empresariales y de trabajo calificado, la capacidad de perseguir opciones innovativas y de calidad, y la actualización de los procesos y de los productos parecen ser elementos cruciales, sea cuando se trata de arraigar un proceso de desarrollo como cuando, en cambio, el objetivo es el de contrarrestar una situación de estancamiento o declinación, de cualquier origen. ¿Pueden las politicas influir sobre la disponibilidad y la eficacia de los mencionados factores? La respuesta parecería incierta en el caso argentino, dadas las frecuentes criticas de la bibliografía en materia: escasa eficacia, falta de coordinación, financiamien-

(21)Por ej., la comercialización de las semillas de soja transgénicas había sido precedida por investigaciones efectuadas con financiamientos públicos (BISANG, 2007; SZTULWARK, 2007). 
tos insuficientes, poca visibilidad y estabilidad. Sin embargo también en Argentina los ejemplos de éxito no faltan: el FONTAR es unánimemente considerado una estructura estable y eficiente, a buen nivel de competencia técnica. En todo caso, hoy ya no se trata de guiar el catching up tecnológico sino, sobre todo, de activar sinergias entre empresas en el interior de la hilera (o entre hileras complementarias), extendiendo el campo de las interacciones en toda su extensión, ampliando y actualizando la gama de los productos. Piénsese otra vez en el ejemplo del sostenimiento acordado en el renacimiento de la industria de las máquinas agrícolas en el centro de la Pampa Humeda, en el distrito de Santa Fe.Otros buenos ejemplos de política industrial presentan todavía el uso de instrumentos tradicionales, tales como las facilitaciones fiscales y crediticias en dirección horizontal, en la emergencia del 2001-2002.

Las enseñanzas que se pueden extraer de la experiencia Argentina son, por lo tanto, más de orden general que puntual: políticas de linkage y de contexto son necesariamente especificas de cada ambiente. En el contexto del redimensionamiento de los fines de la política industrial y del reposicionamiento de la vocación productiva, los ejemplos de éxito son aquellos que han logrado contrarrestar amenazas de desertificación productiva, provenientes de los desafíos de la competencia internacional.

A veces parece que el rol de coordinación, difusión del conocimiento y de control de la organización industrial puede ser directamente desempeñado por las grandes empresas líderes cuyas decisiones de internacionalización o desverticalización, deslocalización, etc, influencian la amplitud y los roles de una red satelital de PyMEs. Estructuras de red que recuerdan a los keiretsu verticales japoneses parecerían emerger del caso Argentino. Por otra parte, las políticas nacionales parecen concientes de los límites y riesgos de sostenibilidad, y de las posibles implicancias negativas en términos sociales y ambientales, de las estrategias implementadas por los grandes operadores para minimizar los costos. La Argentina además parece permanecer alejada de la capacidad de establecer interacciones de policy sobre bases cooperativas o contractuales con la gran empresa, en gran porcentaje bajo control extranjero.

Permìtasenos como final una alusión al rol insustituible de las Universidades públicas, respecto de la investigación de base como, sobre todo, de la formación, actualización y activación de la gran dotación de capital humano del cual dispone el País ${ }^{(22)}$.

\section{Bibliografía}

ANLLÓ, Guillermo; LUGONES, Gustavo; PEIRANO, Fernando. La innovación en la Argentina post-devaluación. Antecedentes previos y tendencias al futuro. En: KOSACOFF, Bernardo. (Ed.). Crisis, recuperación y nuevos dilemas. La economía argentina 2002-2007. Buenos Aires: Cepal, 2007. p. 261-306.

ASIAIN, Andrés. Re-tensiones a la exportación, una mirada más allá de la cuestión fiscal. Realidad Económica, n. 236, 2008. p. 8-30.

(22) Para el caso Argentino, ver la reseña crítica de Langer (2008). 
BARUJ, Gustavo; RAMOS, Adrián; KOSACOFF, Bernardo. Las políticas de promoción de la competitividad en la Argentina. Principales instituciones e instrumentos de apoyo y mecanismos de articulación público-privada. Buenos Aires: Cepal, 2008.

BIANCHI, Patrizio. Che cosa impariamo dalla crisi argentina? LIndustria, XXIII, 2002. p. 5-28.

; LABORY, Sandrine; PACI, Daniele; PARRILLI, Mario Davide. Small and medium-sized enterprise policies in Europe, Latin America and Asia. En: BIANCHI, P.; LABORY, S. (Eds.) International Handbook on Industrial Policy, Cheltenham, Edward Elgar, 2006. p. 380-402.

BISANG, Roberto. El desarollo agropecuario en las últimas decadas: volver a creer? En: KOSACOFF, B. (Ed.). Crisis, recuperación y nuevos dilemas. La economía argentina 2002-2007. Buenos Aires: Cepal, 2007. p. 380-402.

; GUTMAN, Graciela. Nuevas dinámicas en la producción agropecuaria: un equilibrio peligroso. Revista Encrucijadas, n. 21, p. 1-23, 2003.

CEP. Los exportadores medianos y pequeños en 2003-2006. Síntesis de la Economía Real, Segunda Epoca, n. 56, p. 43-62, 2007.

Performance exportadora reciente de las pequeñas y medianas empresas. Realidad Económica, n. 214, 2005a. p. 61-73.

Substitución de importaciones: una aproximación cuantitativa. Buenos Aires: Centro de Estudios para la Producción, Secretaría de Industria, Comercio y de la Pequeña y Mediana Empresa, Ministerio de Economía y Producción, mimeo, 2005b.

CHUDNOVSKY, Daniel; LÓPEZ, Andrés. La transnacionalización de la economía argentina. Buenos Aires: EUDEBA, mimeo, 2001.

CIMOLI, Mario; PORCILE, Gabriel; PRIMI, Annalisa; VERGARA, Sebastián. Cambio estructural, heterogeneidad productiva y tecnología en América Latina. En CIMOLI, M. (Ed.), Heterogeneidad estructural, asimetrías tecnológicas y crecimiento en América Latina. Santiago: Cepal, 2005. p. 9-39.

DAMILL, Mario. El balance de pagos y la deuda externa pública bajo la convertibilidad. Boletin Techint, n. 303, 2000.

FERNÁNDEZ BUGNA, Cecilia; PORTA, Fernando. El crecimiento reciente de la industria argentina. Nuevo régimen sin cambio estructural. En: KOSACOFF, Bernardo. (Ed.). Crisis, recuperación y nuevos dilemas. La economía argentina 2002-2007. Buenos Aires: Cepal, 2007. p. 107-148.

FUNDACIÓN OBSERVATORIO PyME. Informe 2005-2006. Evolución reciente, situación actual y desafíos futuros de las PyMEs industriales. Buenos Aires: 2006.

GALEANO, Eduardo. Las venas abiertas de America. Montevideo, Catalogos, 1971.

GIARRACCA, Norma; TEUBAL, Miguel; PALMISANO, Tomás. Paro agrario: crónica de un conflito alargado. Realidad Económica, n. 237, 2008. p. 33-54.

HERNANDEZ, Valeria A. El fenomeno económico y cultural del boom de la soja y el empresario innovador. Desarrollo Económico, n. 47, p. 331-365, 2007.

KANTIS, Hugo; FEDERICO, Juan. Crisis y renacimiento emprendedor en la Argentina: evidencias y algunos interrogantes. En: FORCINITO, Karina; BASUALDO, Victoria. (Coord.). Transformaciones recientes en la economía argentina. Tendencias y perspectivas. Buenos Aires: Prometeo Libros, 2007. p. 143-166.

KULFAS, Matías. Las PyMES de cara al futuro. Desarrollo PyME, n. 2, p. 4-7, 2007.

; DUARTE, Marisa; SCHORR, Martín. Asociatividad de micro, pequeñas y medianas empresas y economías de aglomeración en la Argentina: teoría, problemáticas y casos de intervención publica. Realidad Económica, n. 235, p. 29-61, 2008. 
LANGER, Ariel. El sistema científico y las universidades: revisión de teorías y enfoques en América Latina y Argentina. En: RIQUELME, Graciela. (Ed.). Las universidades frente a las demandas sociales y productivas. Buenos Aires, UBA, UnaM, UNMdP, 2008. p. 23-73.

LAVOPA, Alejandro. La Argentina posdevaluación. Un nuevo modelo económico? Realidad Económica, n. 231, p. 48-74, 2007.

LEÓN, Carlos. Fondo Tecnológico Argentino Fontar. Memoria 1995-2007. Buenos Aires: Agencia Nacional de Promoción Científica y Tecnológica, 2008.

LEVY YEYATI, Eduardo; VALENZUELA, Diego. La resurreción. Historia de la poscrisis argentina. Buenos Aires: Editorial Sudamericana, 2007.

MILESI, Dario; MOORI, Virginia; ROBERT, Verónica; YOGUEL, Gabriel. Desarollo de ventajas competitivas: pymes exportadoras exitosas en Argentina, Chile y Colombia. Revista de la Cepal, $\mathrm{n}$. 92, p. 48-74, 2007.

PERES, Wilson. El lento retorno de las políticas industriales en América Latina y el Caribe. Revista de la Cepal, n. 88, p. 71-88, 2006.

SCHORR, Martín. Argentina: la industria que el neoliberalismo nos legó. En: FORCINITO, Karina; BASUALDO, Victoria. (Coord.). Transformaciones recientes en la economía argentina. Tendencias y perspectivas. Buenos Aires: Prometeo Libros, 2007. p. 117-141.

SZTULWARK, Sebastián. Dinámica tecnológica y especialización productiva en la agricultura argentina. En: FORCINITO, Karina; BASUALDO, Victoria. (Coord.). Transformaciones recientes en la economía argentina. Tendencias y perspectivas. Buenos Aires: Prometeo Libros, 2007. p. 99-114.

\section{APÉNDICE: LAS POLÍtICAS INDUSTRIALES PARA LAS PEQUEÑAS Y MEDIANAS EMPRESAS EN Argentina}

Del conjunto de las políticas industriales argentinas, aquellas destinadas en particular a las pequeñas y medianas empresas se remontan a una época bastante reciente: el 1995, año en que se adopta la primera Ley PyME, mientras que en 1997 se asiste a la creación de la Secretaría de la Pequeña y Mediana Empresa (SEPyME), transformada sucesivamente en la Subsecretaría de la Pequeña y Mediana Empresa y Desarrollo Regional (SSEPyMEyDR). Aunque las políticas económica e industrial han tenido un profundo impacto, que ha influenciado también a las pequeñas y medianas empresas, es a partir de esa fecha que comienzan a delinearse en el interior del profundo e importante proceso de reforma de la economía argentina una relevancia específica y una atención dirigida hacia este segmento ${ }^{(23)}$. Por tanto es a partir de esa fecha que comienza nuestro análisis.

Siguiendo la fuente más completa y reciente a nuestra disposición (BARUJ, RAMOS, KOSAKOFF, 2008), distinguiremos entre políticas de promoción de la Innovación, de las Exportaciones, de las Inversiones e Inversiones Externas Directas (IED) y Capacidades en general de las empresas.

(23) Según Bianchi, Labory, Paci y Parrilli (2006), Argentina representa el país que ha desarrollado mayormente la política industrial hacia las PyMEs. Para dar una idea del empeño, reportamos aquí las cifras presentadas por el entonces Subsecretario de la SEPyME: "Estas políticas han permitido que 15.000 PyMEs accedieran al crédito a tasas subsidiadas, 9.000 obtuvieran diversas formas de financiamiento a través del sistema de garantías reciprocas, 900 se financiaran a largo plazo para inversión - además de 1.000 subsidios otorgados para reconversión productiva y certificación de normas de calidad —, más de 300 participaran de proyectos asociativos agrupadas en clusters, otras 1.000 pudieran capacitar a su personal con un subsidio que cubrió el 100\% de dichos gastos, y cerca de 4.000 pequeñas y medianas empresas participaran en acciones tendientes a estimular sus exportaciones, entre otras." (KULFAS, 2007). 
El marco de las Políticas para la innovación se desarrolla a partir de la Ley 23.877 de Promoción y Fomento de la Innovación Tecnológica (1990), inspirada por un planteo fuertemente horizontal y que intentaba sostener la demanda de tecnología de las empresas, a las que ya favorecía el tipo de cambio sobrevaluado, para adquirir en el exterior bienes de capital que incorporasen esas nuevas tecnologías. La creación de la Agencia Nacional de Promoción Científico y Tecnológica siguió al lanzamiento del Programa de Modernización Tecnológica en 1997, implementado en 1994 con un financiamiento del Banco Interamericano de Desarrollo. Es éste un organismo descentralizado dependiente de la Secretaría de Ciencia, Tecnología e Innovación Productiva (SeCTIP) del Ministerio de Educación, Ciencia y e Tecnología, cuyo accionar se articula a través del Fondo Tecnológico Argentino (FONTAR), el Fondo para la Investigación Científica y Tecnológica (FONCYT) y, más recientemente, del Fondo Fiduciario para la Promoción de la Industria del Software (FONSOFT).

Los instrumentos para sostener los proyectos de mejoras de la productividad de las empresas son créditos, subsidios e incentivos fiscales. Hasta el presente no se ha procedido con la reforma iniciada con la Ley 25.467 que instituía el Sistema Nacional de Ciencia, Tecnología e Innovación, mientras que el Instituto Nacional de Tecnología Agropecuaria (INTA) y el Instituto Nacional de Tecnología Industrial (INTI) han mantenido un rol de asistencia técnica a las empresas (agrícolas o industriales), sin competencias relevantes en el campo de la innovación.

Entre las actividades de estos organismos se destacan las del FONTAR, que ha llegado a financiar alrededor de 300 proyectos al año en el 2006 y en el 2007, con un 85\% de las contribuciones desembolsadas destinado a PyMEs. Entre las iniciativas más estudiadas, se señala red que incluye alrededor de 200 Unidades de Vinculación Tecnológica (UVT) que operan como nexo entre universidades y centros de investigación y empresas ${ }^{(24)}$. Últimamente se ha lanzado una nueva línea de financiamiento destinada a grupos de empresas, centros de investigación y formación superior ligados a un aglomerado productivo (Programa de Desarrollo de Complejos Productivos Regionales). La idea está inspirada en la experiencia distritual italiana, con miras a favorecer la cooperación entre empresas sobre base territorial, en áreas de alta concentración de PyMEs, pero intentando superar los límites que presentan en las áreas periféricas los modelos de desarrollo local propios de economías más avanzadas, sobre todo basadas en la estabilidad política, institucional y económica. Es todavía prematuro llegar a una conclusión sobre el programa, para el que han sido seleccionados 11 proyectos ${ }^{(25)}$, pero las impresiones de quienes han participado directamente hablarían de pocos casos exitosos, y muy a menudo de "sobrevivencia a niveles escasos de productibilidad y rentabilidad" (KULFAS, DUARTE, SCHORR, 2008).

En las Políticas para la Exportación el rol principal ha sido asumido por la política monetaria: al principio un cambio sobrevaluado ha impulsado la adopción de tecnologías capital intensive, luego la devaluación ha favorecido la producción de mercaderías

(24) Evaluaciones de la experiencia del Fontar en la síntesis de Anlló, Lugones y Peirano (2007) y los trabajos en ella citados. Ver también León (2008).

(25) Para dar una idea de las áreas y las industrias involucradas, se va del área vitivinícola de la Región Andina, a la apicultura en el Noroeste, a las forestas de Misiones, al cluster metalmecánico de Olavarría, a las maquinarias agrícolas del Centro, a el aguacultura de Río Negro. 
destinadas al mercado interno. Los instrumentos de política industrial derivan de los años de la convertibilidad, y están orientados a impulsar exportaciones a través del trato privilegiado hacia las empresas exportadoras, que en su mayoría son grandes empresas de sectores tradicionales ${ }^{(26)}$.

Estos instrumentos están establecidos por ley y administrados en gran parte por la Subsecretaría de Política y Gestión Comercial que depende de la Secretaría de Industria, Comercio y del Ministerio de Economía y Producción.

Otras instituciones, entre ellas la Fundación EXPORT-AR, organizaciones de composición mixta, pública-privada sin fines de lucro, y la mencionada SSEPyMEyDR, contribuyen en la asistencia técnica, pero su rol en el conjunto es limitado desde el punto de vista de las financiaciones involucradas.

Encontramos dos "regímenes" interesantes, al menos desde un punto de vista histórico-político. En base al primero, como consecuencia al acuerdo sobre la materia entre Argentina y Brasil (1995), se eliminaron los impuestos sobre las importaciones de autopartes y vehículos con la condición de compensarlos con exportaciones del mismo monto (en lo que respecta a vehículos) o superiores al 20\% (en lo que respecta a las autopartes). El segundo se remonta a tiempos (afortunadamente) más lejanos y es referido a la completa exención fiscal para las empresas localizadas en Tierra del Fuego, Antártica e Islas del Atlántico Sur, motivado por preocupaciones de naturaleza estratégica en los tiempos del régimen militar.

La política de atracción - "indiscriminada" según algunos comentaristas como Damill (2000), Chudnovsky y López (2001), Schorr (2007) - de las Inversiones Externas Directas (IED) de los años 90 ha llevado a la extranjerización de importantes servicios públicos, y de los sectores minero, agroindustrial y energético. La mayor parte de las inversiones se ha dirigido a la compra de empresas nacionales (públicas y privadas) por parte de capitales extranjeros, aunque la proporción de las inversiones greenfields alcanza de todos modos el $40 \%$. Hay que destacar que este flujo se ha interrumpido bruscamente con el default del 2001 y, antes del estancamiento del 2008, estaba remontando, aunque lentamente en relación a la velocidad de la "resurrección" argentina.

La principal institución que opera en este campo es la Subsecretaría de Industria, a la cual se suman la SSEPyMEyDR, el Banco de Inversión y Comercio Exterior (BICE), el Banco de la Nación Argentina y la recientemente (2006) instituida Agencia Nacional de Desarrollo de Inversiones (ADI). Los instrumentos utilizados consisten en créditos y desgravaciones impositivas, frecuentemente usados cuando se trata de medidas sectoriales, como las mencionadas anteriormente relacionadas a las industrias forestales, del tabaco, automovilística, del software.

Finalmente, entre las instituciones dedicadas a la promoción de las Capacidades endógenas de las empresas, el rol principal es de nuevo asumido por la SSEyDR, con un impacto relevante en términos de PyMEs involucradas, pero muy discutido en términos de resultados efectivos sobre la competitividad de las empresas.

(26) Basta pensar que, según fuentes oficiales, en el 2006 las 921 mayores empresas argentinas han efectuado el $92.2 \%$ de las exportaciones del País (CEP, 2007). 
Es interesante destacar, como anteriormente hemos hecho con referencia a la maquinaria agroindustrial, que en Argentina existe un conjunto de intervenciones que bien podríamos definir de política industrial, de naturaleza privada, que reemplazan a las escasas visibilidad, accesibilidad y estabilidad de los programas públicos, además de las bien conocidas (y no exclusivamente argentinas) dificultades burocráticas y desconfianza de parte de las empresas. No sorprende, además, que muchas iniciativas tengan un marcado carácter sectorial, dada la parcial deserción de las instituciones públicas en este campo. Hay que destacar además que las financiaciones son frecuentemente de proveniencia externa, de fundaciones y organismos internacionales de carácter público o privado. Entre estos tipos de programas resaltan aquellos lanzados por la Unión Industrial Argentina (UIA), y los de la organización Techint, orientados a mejorar la competitividad de las PyMEs relacionadas al grupo, proveedores o clientes. 